\title{
Clinical experience with tigecycline in the treatment of hospital-acquired pneumonia caused by multidrug resistant Acinetobacter baumannii
}

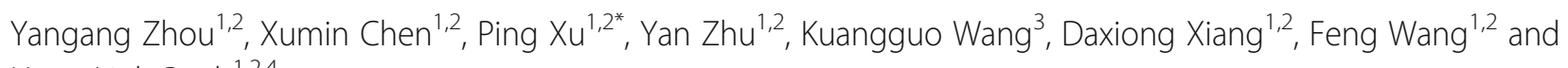
Hoan Linh Banh ${ }^{1,2,4}$

\begin{abstract}
Background: Tigecycline, with broad in vitro antibacterial activity, has been widely used off-label for nosocomial pneumonia caused by multi-drug resistant Acinetobacter baumannii (MDRAB). However, many concerns have been raised about the efficacy of tigecycline treatment as the inconsistent results from previous clinical studies.

Methods: This retrospective study evaluated the outcome of adult patients with monomicrobial MDRAB nosocomial pneumonia treated with tigecycline between 2015 and 2017. Results.

A total of 77 patients was eligible for this study, and the overall clinical success and 30-day survival rates were 70.03 and $70.13 \%$, respectively, however, the microbiological eradication rate was relatively low (48\%). Multivariate analysis indicated that shorter duration of tigecycline use associated with increased clinical failure, whereas higher CURB65 scores, mechanical ventilation and tigecycline resistant to MDRAB have significant association with 30-day mortality.

Conclusions: Our results suggest that tigecycline is one of the potential choices for the treatment of hospital-acquired pneumonia caused by MDRAB, especially with a MIC $\leq 2 \mathrm{mg} / \mathrm{L}$. In addition, a longer duration of tigecycline treatment may be required to insure better clinical outcomes.
\end{abstract}

Keywords: Tigecycline, Multi-drug resistance, Acinetobacter baumannii, Hospital-acquired pneumonia

\section{Backgrounds}

Pneumonia caused by multidrug-resistant Acinetobacter baumannii (MDRAB) is a challenge in nosocomial infection as it has become resistant to most antibiotics, including penicillins, cephalosporins, aztreonam, fluoroquinolones, aminoglycosides and even carbapenems, resulting in limited antibiotic options for treatment $[1,2]$.

Tigecycline, the first member of the glycylcycline class of antimicrobial agents, has been approved by the Food and Drug Administration (FDA) for the treatment of complicated skin and skin-structure infections, complicated

\footnotetext{
* Correspondence: xuping1109@csu.edu.cn

'Department of Pharmacy, the Second Xiangya Hospital, Central South University, No.139 Middle Renmin Road, Changsha, Hunan 410011, People's Republic of China

${ }^{2}$ Institute of Clinical Pharmacy, Central South University, Changsha, People's Republic of China

Full list of author information is available at the end of the article
}

intra-abdominal infections and community-acquired pneumonia [3]. Because of its expanded spectrum of in vitro antibacterial activity, and high sensitivity to MDRAB, tigecycline has been widely used off-label for nosocomial pneumonia caused by MDRAB [4, 5].

According to previous studies, tigecyclineis no better than standard antimicrobial agents for the treatment of various infections caused by MDRAB, and mortality from all causes was even higher in the tigecycline group [6-9]. However, since most of these studies focused on the multisite infections with more than one pathogen, it is difficult to establish the effect of tigecycline as a single agent on MDRAB with so many confounding factors. As a result, it is unclear whether tigecycline is an effective option for treating pneumonia caused by MDRAB. Therefore, we conducted a retrospective study with monomicrobial MDRAB pneumonia to assess the effectiveness of tigecycline for the

(C) The Author(s). 2019 Open Access This article is distributed under the terms of the Creative Commons Attribution 4.0 International License (http://creativecommons.org/licenses/by/4.0/), which permits unrestricted use, distribution, and 
treatment of MDRAB nosocomial pneumonia, and to identify the predictors of treatment success.

\section{Methods}

\section{Study design, subjects and treatments}

This was a retrospective study performed in the second Xiangya Hospital of Central South University (Changsha, China), a tertiary-care teaching hospital with more than 3000 beds, between January 2015 and December 2017. The study received approval from the Second Xiangya Hospital of Central South University Ethics Committee. All hospitalized patients age $\geq 18$ years who received tigecycline for the treatment of hospital-acquired pneumonia (HAP) involving monomicrobial MDRAB were included in this study. Tigecycline treatment was at least 5 days, with a $100 \mathrm{mg}$ loading dose followed by $50 \mathrm{mg}$ administered intravenously every $12 \mathrm{~h}$. Data included demographic characteristics, medical history, clinical and laboratory findings, diagnostic imaging and type of treatment and outcome were extracted from the electronic patient medical records. The primary study outcomes were clinical and microbiologic success rates, and we also investigated the factors associated with clinical failure and mortality.

\section{Definition}

Hospital-acquired pneumonia (HAP) was defined as pneumonia that occurred $48 \mathrm{~h}$ or more after admission. Pneumonia was diagnosed according to the American Thoracic Society Guidelines 2005, which consists of a new or progressive infiltrate on chest X-ray with two or more of the following clinical characteristics: new onset of fever $\left(\geq 38^{\circ}\right.$ C) or hypothermia $\left(<35.5^{\circ} \mathrm{C}\right)$, leukocytosis (leukocyte count $>10,000$ cells $/ \mathrm{mm}^{3}$ ) or leukopenia (leukocyte count< 4000 cells/mm3), oxygen desaturation, and positive purulent sputum [10]. Monomicrobial MDRAB pneumonia was defined as a positive sputum or tracheal aspirate culture of only MDRAB from 1 week before up to 1 week after the initiation of first dose of tigecycline, and there was no other infection at the start of the treatment. Severity of pneumonia was based on a CURB-65 score which was recorded within $48 \mathrm{~h}$ of the administration of first dose of tigecycline. All cause 30-day mortality was defined as death during 30 days of treatment with tigecycline. Clinical resolution of pneumonia at the end of treatment was defined as improvement of the subsequent chest X-ray, or partial or complete resolution of signs and symptoms of infection and improvement or resolution of laboratory tests such as white blood cell count (decrease to normal), C-reactive protein (CRP) (decrease to normal or by $\geq 30 \%$ ), and procalcitonin (PCT) (decrease to normal or by $\geq 80 \%$ ) [11]. Delayed tigecycline treatment was defined as 3 days delay of tigecycline use after the detection of airway MDRAB isolates [12].

\section{Microbiology}

The antibiotic susceptibility profiling of isolates had been performed (except tigecycline and cefoperazone sulbactam) using a BD Phoenix-100 automated microbiology system (Diagnostic Systems, Sparks, MD) [13]. The results were interpreted according to the breakpoints suggested by the Clinical and Laboratory Standards Institute (CLSI) 2016. Susceptibility to tigecycline was determined using disk diffusion method Standard of antibiotics Susceptibility Test (Kirby-Bauer Method), and the diameter of inhibition zone $\geq 16 \mathrm{~mm}, 13-15 \mathrm{~mm}, \leq 12 \mathrm{~mm}$ interpreted as susceptible, intermediately resistant and resistant, respectively. MDRAB was defined as A. baumonnii resistance to at least three of the following classes of antibiotics: aminoglycosides, antipseudomonal penicillins, carbapenems, cephalosporins and quinolones. Microbiological eradication was defined as the absence of MDRAB from all subsequent respiratory cultures. Bacteremia was defined as one or more positive blood culture for Acinetobacter baumannii during tigecycline treatment.

\section{Statistical methods}

All statistical analyses were performed using the Statistical Package for the Social Sciences for Windows (Version 18.0; SPSS Inc., Chicago, IL, USA). Categorical variables were compared using chi-square test. Continuous variables were tested for normality of distributions by Kolmogorove-Smirnov test, and then compared by the Mann-Whitney $U$ test. Odd ratios (ORs) and 95\% confidence interval $(\mathrm{CI})$ were calculated. Variables with a $p$ value $<0.05$ in univariate analysis were included in a logistic regression model for multivariate analysis. All tests were two-tailed, and a $p$ value $<0.05$ was considered statistically significant in multivariate analysis.

\section{Result}

Patients, demography and concomitant diseases

There were 598 patients received tigecycline between 2015 and 2017. A total of 77 patients with monomicrobial MDRAB were identified (Fig. 1), patient demographics and clinical features are summarized in Table 1 . The mean age was 62.1 years and $52(67.53 \%)$ were male. The major comorbidities were chronic obstructive pulmonary disease (COPD) (31.17\%) and hypertension (22.08\%) and 16 patients had surgery (20.78\%).

\section{Clinical characteristics of infection and treatment}

The mean CURB65 score was 2.02. CURB65 severity score for pneumonia severity is summarized in Table 2 [14]. Almost half of the patients, 48 (62.34\%), were on mechanical ventilation during tigecycline treatment. Almost all of the cultures with only MDRAB isolated were from airway specimens with only 7 of cultures are from blood samples. Among the 77 MDRAB isolates, 52 


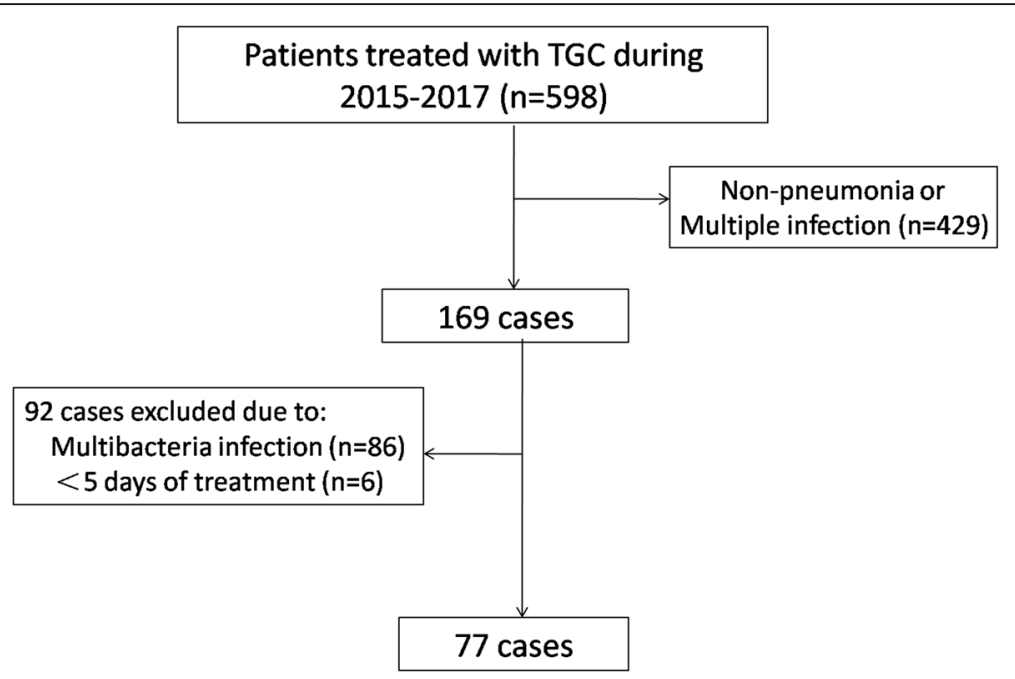

Fig. 1 Allocation of MDR/XDRAB pneumonia patients included. MDR/XDRAB = multidrug-resistant and extensively drug-resistant Acinetobacter baumannii; TGC = tigecycline.

$(67.53 \%)$ and $20(25.97 \%)$ were resistant or intermediately resistant to sulbactam, and were all resistant to carbapenems, fluoroquinolones and amikacin. Only 6 isolates (7.79\%) were resistant, and 29 (37.66\%) were intermediate resistant to tigecycline. No resistance to colistin was found. The mean duration of tigecycline use was 11.69 days, and treatment was discontinued in 3 patients on day 5 of treatment due to diarrheal and the elevator of serum total bilirubin level. There were 71 patients (92.21\%) received combination therapy with tigecycline. Cefoperazone sulbactam was the most common co-administered agents $(64 / 71,90.14 \%)$, followed by carbapenems and fluoroquinolones (4/71, 5.63\%); 21 patients (27.27\%) had delayed tigecycline treatment (Table 1).

\section{Microbiological outcomes}

There were 50 patients that had subsequent follow-up respiratory tract cultures after the tigecycline treatment, and $24(48 \%)$ of them had airway eradication from MDRAB.

\section{Clinical outcomes}

The all cause 30-day mortality rate was (29.87\%). Only 58 patients had follow-up chest radiographs after tigecycline treatment with $21(36.21 \%)$ patients showed improvement and $57(70.03 \%)$ patients had clinical resolution of pneumonia.

\section{Risk factors for clinical resolution of tigecycline treatment for MDRAB pneumonia}

In univariate analysis, patients without clinical resolution of pneumonia had higher CURB65 scores, higher rates of ventilator associated pneumonia (VAP) and bacteremia caused by MDRAB, delayed tigecycline treatment, shorter duration of tigecycline use and lower ratio of airway eradication of MDRAB when comparing to the patients with clinical resolution $(p<0.05)$ (Table 3$)$. Variables with a $p$ value $<0.05$ were included in multivariate analysis, and the independent predictor for failure of clinical resolution was the shorter duration of tigecycline use (Table 4).

\section{Risk factors for the 30-day mortality}

In univariate analysis, patients with 30-day mortality had higher CURB65 scores, higher resistance rate to tigecycline, VAP and bacteremia caused by MDRAB, and shorter duration of tigecycline use when comparing to the survival patients $(p<0.05)$ (Table 5$)$. Variables with a $p$ value $<0.05$ were included in multivariate analysis, and the independent predictors for 30-day mortality were higher CURB65 score, VAP and tigecycline resistance (Table 6).

\section{Discussion}

This study evaluated the outcomes of 77 patients with monomicrobial MDRAB nosocomial pneumonia treated with tigecycline. The overall clinical resolution and 30-day survival rates were 70.0 and $70.1 \%$, respectively. The microbiological eradication rate was $48.0 \%$. Based on previous studies, the clinical resolution of tigecycline treatment was between 45.2 and $84.0 \%$ [15-17], and the difference between studies may be due to the severity of illness, since the clinical success rate were relatively lower in intensive care patients who had higher APACHEII scores. Similar to previous studies, positive microbiological response rates were lower when compared with successful clinical outcome in this study. The concerns were the inconsistency of clinical success and microbiologic response in previous studies may not be attributed to tigecycline, rather it is due to misdiagnosis. Curcio [18] et al. found that the non-bronchoalveolar lavage method 
Table 1 Characteristics of the 77 patients of tigecycline-treated pneumonia involving multidrugresistant Acinetobacter baumannii (MDRAB)

\begin{tabular}{|c|c|}
\hline Characteristics & values \\
\hline \multicolumn{2}{|l|}{ Demographic parameters } \\
\hline Age, mean $\pm S D$, years & $62.1 \pm 17.0$ \\
\hline Male/Female & $52 / 25$ \\
\hline \multicolumn{2}{|l|}{ Comorbidities } \\
\hline Hepatic dysfunction & $2(2.6 \%)$ \\
\hline Renal insufficiency & $7(9.1 \%)$ \\
\hline Chronic pulmonary disease & $24(31.2 \%)$ \\
\hline Heart disease & $14(18.2 \%)$ \\
\hline Hypertension & $17(22.1 \%)$ \\
\hline Diabetes mellitus & $6(7.8 \%)$ \\
\hline Immune compromise & $5(6.5 \%)$ \\
\hline multiple organ failure & $3(3.9 \%)$ \\
\hline Malignancy & $8(10.4 \%)$ \\
\hline Surgery & $16(20.8 \%)$ \\
\hline \multicolumn{2}{|l|}{ Clinical conditions } \\
\hline CURB65 score, mean \pm SD & $2.0 \pm 1.0$ \\
\hline Mechanical ventilation & $48(62.3 \%)$ \\
\hline \multicolumn{2}{|c|}{ Susceptibility tests of initial airway MDRAB isolates } \\
\hline With sensitive to tigecycline & $42(54.5 \%)$ \\
\hline With sensitive to sulbactam & $7(9.1 \%)$ \\
\hline \multicolumn{2}{|l|}{ Tigecycline treatment } \\
\hline Duration, mean $\pm S D$, days & $11.69 \pm 6.11$ \\
\hline Combination therapy & $71(92.2 \%)$ \\
\hline With sulbactam & $64(90.1 \%)$ \\
\hline With carbapenems & $4(5.6 \%)$ \\
\hline With amikacin & $1(1.4 \%)$ \\
\hline With fluoroquinolones & $4(5.6 \%)$ \\
\hline Delayed tigecycline treatment & $21(27.3 \%)$ \\
\hline
\end{tabular}

Abbreviations: $S D$ standard deviation

for microbiology sampling predicts poor clinical outcomes, which means that using bronchoalveolar lavage (BAL) may result in higher accuracy in identifying microbiologic organisms and reduce the risk of misdiagnosis. This study only included cultures positive for monomicrobial MDRAB, and among the patients with follow-up sputum cultures, the clinical success rate was significantly higher in the group of microbiological eradication (21/24,
$87.5 \%$ vs $20 / 33,60.6 \% ; p=0.026)$. Hence, the lower microbiological response in this study may be due to the strict inclusion criteria compared to previous studies, since only complete eradication of MDRAB was defined as microbiological success. The clinical success was higher as the definition was less strict with both partial and complete resolution of clinical symptoms. This finding has also been shown in other clinical studies evaluating antimicrobials for treatment of hospital-acquired pneumonia (HAP) or ventilator-associated pneumonia (VAP) which made it difficult to directly compare the effect of different antibiotics [19]. Thus, it is essential to standardize terms used in enrolment criteria, endpoints, microbiological and clinical cure in future studies to better understand the results.

To use tigecycline appropriately, predictors for clinical success and 30-day mortality in patients with monomicrobial MDRAB nosocomial pneumonia were investigated in this study. The multivariate analyzes showed that the prolonged tigecycline usage was significantly associated with clinical resolution, whereas higher CURB65 scores, mechanical ventilation and tigecycline resistant to MDRAB have significant association with 30-day mortality, and these results may help to explain previous results. Many studies compared the efficacy of tigecycline in the treatment of MDRAB with other antibiotics, and tigecycline was not recommended as first line treatment for MDRAB because of the higher mortality and lower microbiological success, especially for bacteremia $[6-8,20]$. However, further analysis showed that the increased mortality rate of tigecycline is significant higher only among those with $\mathrm{MIC}>2 \mathrm{mg} / \mathrm{L}$, but not for those with $\mathrm{MIC} \leq 2 \mathrm{mg} / \mathrm{L}$. Kim et al. [21] investigated the effectiveness of tigecycline-based versus colistin-based therapy for treatment of MDRAB pneumonia. There was no difference between the groups with respect to clinical outcomes, microbiological success and 30-day mortality, in which most MDRAB isolates showed a tigecycline MIC $\leq 2 \mathrm{mg} / \mathrm{L}$. Hence, the sensitivity of tigecycline might be the key when choosing the regimen against HAP caused by monomicrobial MDRAB. With respect to mortality, MDRAB with $\mathrm{MIC}>2 \mathrm{mg} / \mathrm{L}$ may not be appropriate to treat MDRAB bacteremia with tigecycline $50 \mathrm{mg}$ every $12 \mathrm{~h}$ since tigecycline achieve low plasma concentration at this dose. Of note, the present study showed that longer treatment duration may produce better clinical outcomes; this may due to the prolonged time needed for bacteriostatic agent to

Table 2 CURB65 Severity for Pneumonia

\begin{tabular}{lll}
\hline Score & Risk & Disposition \\
\hline 0, or 1 & $1.5 \%$ mortality & Outpatient care \\
2 & $9.2 \%$ mortality & Inpatient vs observation admission \\
$\geq 3$ & $22 \%$ mortality & Inpatient admission with consideration for ICU admission with score of 4 or 5 \\
\hline
\end{tabular}


Table 3 Univariate analysis of the predictors for failure in clinical resolution (CR)

\begin{tabular}{|c|c|c|c|c|}
\hline Variables & With $C R^{a} n=57$ & Without $C R^{a} n=20$ & OR $(95 \% \mathrm{Cl})$ & $p$ \\
\hline \multicolumn{5}{|l|}{ Demographic parameters } \\
\hline Age, year & $59.4(18.9)$ & $63.09(16.3)$ & & 0.464 \\
\hline Female gender & $16(28.1 \%)$ & $9(45.0 \%)$ & $0.48(0.17-1.37)$ & 0.164 \\
\hline \multicolumn{5}{|l|}{ Comorbidities } \\
\hline Hepatic dysfunction & $2(3.5 \%)$ & $0(0.0 \%)$ & $0.73(0.64-0.84)$ & 1.000 \\
\hline Renal insufficiency & $6(10.5 \%)$ & $1(5.0 \%)$ & $2.24(0.25-19.80)$ & 0.669 \\
\hline Chronic pulmonary disease & $14(24.6 \%)$ & 7 (35.0\%) & $0.60(0.20-1.82)$ & 0.367 \\
\hline Heart disease & $11(19.3 \%)$ & $2(10.0 \%)$ & $2.15(0.43-10.68)$ & 0.495 \\
\hline Diabetes mellitus & $3(5.3 \%)$ & $1(5.0 \%)$ & $1.06(0.10-10.77)$ & 1.000 \\
\hline Immune compromise & $3(5.3 \%)$ & $2(10.0 \%)$ & $0.50(0.08-3.23)$ & 0.600 \\
\hline Malignancy & $5(8.8 \%)$ & $2(10.0 \%)$ & $0.86(0.15-4.86)$ & 1.000 \\
\hline Hypoproteinemia & $1(1.8 \%)$ & $1(5.0 \%)$ & $0.34(0.20-5.69)$ & 0.455 \\
\hline Surgery & $11(19.3 \%)$ & $5(25.0 \%)$ & $0.72(0.22-2.34)$ & 0.749 \\
\hline \multicolumn{5}{|l|}{ Clinical conditions } \\
\hline CURB65 score, mean \pm SD & $2.4(0.6)$ & $1.9(1.1)$ & & 0.006 \\
\hline Mechanical ventilation & $31(64.6 \%)$ & $17(85.0 \%)$ & $0.21(0.06-0.80)$ & 0.015 \\
\hline Albumin & $31.7(5.7)$ & $32.2(4.0)$ & & 0.512 \\
\hline \multicolumn{5}{|l|}{ Microbiology } \\
\hline Bacteremia & $2(3.5 \%)$ & $5(25.0 \%)$ & $0.11(0.02-0.62)$ & 0.011 \\
\hline Tigecycline resistance & 33 (57.9\%) & $9(45.0 \%)$ & $1.68(0.60-4.69)$ & 0.319 \\
\hline Airway eradication of MDRAB & $21(51.2 \%)$ & $3(18.8 \%)$ & $4.55(1.13-18.39)$ & 0.026 \\
\hline \multicolumn{5}{|l|}{ Tigecycline treatment } \\
\hline Duration, days & $8.4(3.7)$ & $12.9(6.4)$ & & 0.001 \\
\hline \multicolumn{5}{|l|}{ Combination therapy } \\
\hline With sulbactam & $49(86.0 \%)$ & $15(75.0 \%)$ & $2.04(0.58-7.19)$ & 0.304 \\
\hline With carbapenems & $3(5.3 \%)$ & $1(5.0 \%)$ & $1.06(0.10-10.77)$ & 1.000 \\
\hline With amikacin & $2(3.6 \%)$ & $1(5.0 \%)$ & $0.70(0.06-8.21)$ & 1.000 \\
\hline With fluoroquinolones & $4(7.0 \%)$ & $0(0.0 \%)$ & $0.73(0.63-0.84)$ & 0.568 \\
\hline Delayed tigecycline treatment & 19 (33.3\%) & $2(10.0 \%)$ & $4.50(0.86-13.31)$ & 0.044 \\
\hline
\end{tabular}

Abbreviations: $O R$ odd ratio, $C l$ confidence interval, $M D R A B$ multidrugresistik Acinetobacter baumannii

${ }^{a}$ Categorical data are no.(\%) of subject, continuous data are expressed as mean (standard deviation)

Table 4 Multivariate analysis of the predictors for failure in clinical resolution

\begin{tabular}{lll}
\hline Variables & Adjusted OR $(95 \% \mathrm{Cl})$ & $p$ \\
\hline CURB65 score & $0.65(0.35-1.23)$ & 0.187 \\
Mechanical ventilation & $0.33(0.07-1.59)$ & 0.165 \\
Bacteremia & $0.21(0.02-2.46)$ & 0.213 \\
Airway eradication of MDRAB & $1.45(0.63-3.34)$ & 0.382 \\
Duration of Tigecycline treatment & $1.23(1.02-1.48)$ & 0.031 \\
Delayed tigecycline treatment & $3.60(0.62-20.90)$ & 0.153
\end{tabular}

Abbreviations: $O R$ odd ratio, $C l$ confidence interval, $M D R A B$ multidrugresistant Acinetobacter baumannii

${ }^{a}$ Categorical data are $\mathrm{n}(\%)$ of subject, continuous data are expressed as mean (standard deviation) completely eradicate the bacteria. Since tigecycline is a bacteriostatic agent, it is possible that a longer duration of treatment is required to treat MDRAB.

Many studies investigated the effect of albumin on antibacterial therapy, especially in critical ill patients. The change of albumin level may significantly affect the pharmacokinetics of antibiotics, subsequently lead to altered antibacterial effect $[22,23]$. Sujata and Tasbakan [24] found that higher albumin was associated with better clinical and microbiological responses to therapy, the results from this study, however, did not show this relationship, it may because only two patients in the study had hypoalbuminemia. As a result, the difference between two groups was not detected.

For all the MDRAB isolates in this study the non-susceptible rate to tigecycline was $42.9 \%$ (33/77) 
Table 5 Univariate analysis of the predictors for the 30-day mortality

\begin{tabular}{|c|c|c|c|c|}
\hline Variables & Death $^{a} n=23$ & Survival $^{a} n=54$ & OR $(95 \% \mathrm{Cl})$ & $p$ \\
\hline \multicolumn{5}{|l|}{ Demographic parameters } \\
\hline Age, year & $63.0(18.5)$ & $61.8(16.5)$ & & 0.644 \\
\hline Female gender & $9(39.1 \%)$ & $16(29.6 \%)$ & $1.53(0.55-4.24)$ & 0.415 \\
\hline \multicolumn{5}{|l|}{ Comorbidities } \\
\hline Hepatic dysfunction & $0(0.0 \%)$ & $2(3.7 \%)$ & $0.69(0.60-0.81)$ & 1.000 \\
\hline Renal insufficiency & $1(4.3 \%)$ & $6(11.1 \%)$ & $0.36(0.41-3.21)$ & 0.667 \\
\hline Chronic pulmonary disease & $6(26.1 \%)$ & $15(27.8 \%)$ & $0.92(0.30-2.78)$ & 0.879 \\
\hline Heart disease & $2(8.7 \%)$ & $11(20.4 \%)$ & $0.37(0.08-1.83)$ & 0.323 \\
\hline Diabetes mellitus & $2(8.7 \%)$ & $2(3.7 \%)$ & $2.48(0.33-18.75)$ & 0.578 \\
\hline Immune compromise & $2(8.7 \%)$ & $3(5.6 \%)$ & $1.62(0.25-10.40)$ & 0.632 \\
\hline Malignancy & $2(8.7 \%)$ & $5(9.3 \%)$ & $0.93(0.17-5.20)$ & 1.000 \\
\hline Hypoproteinemia & $0(0.0 \%)$ & $2(3.7 \%)$ & $0.69(0.60-0.81)$ & 1.000 \\
\hline Surgery & $5(21.7 \%)$ & $11(20.4 \%)$ & $1.09(0.33-3.58)$ & 1.000 \\
\hline \multicolumn{5}{|l|}{ Clinical conditions } \\
\hline CURB65 score, mean \pm SD & $2.7(1.1)$ & $1.7(0.8)$ & & 0.001 \\
\hline Mechanical ventilation & $21(91.3 \%)$ & $27(50.0 \%)$ & $10.5(2.24-49.24)$ & 0.001 \\
\hline Albumin & $32.2(4.9)$ & $32.1(4.3)$ & & 0.916 \\
\hline \multicolumn{5}{|l|}{ Microbiology } \\
\hline Bacteremia & $5(21.7 \%)$ & $2(3.7 \%)$ & $7.22(1.29-40.54)$ & 0.022 \\
\hline Tigecycline resistance & $16(69.6 \%)$ & 19 (35.2\%) & $0.24(0.08-0.68)$ & 0.006 \\
\hline Airway eradication of MDRAB & $5(27.8 \%)$ & $19(48.7 \%)$ & $0.40(0.12-1.35)$ & 0.137 \\
\hline \multicolumn{5}{|l|}{ Tigecycline treatment } \\
\hline Duration, days & $10.0(6.6)$ & $12.4(5.8)$ & & 0.014 \\
\hline \multicolumn{5}{|l|}{ Combination therapy } \\
\hline With sulbactam & 19 (82.6\%) & $45(83.3 \%)$ & $0.95(0.26-3.47)$ & 1.000 \\
\hline With carbapenems & $1(4.3 \%)$ & $3(5.6 \%)$ & $0.77(0.08-7.84)$ & 1.000 \\
\hline With amikacin & $1(4.3 \%)$ & $2(3.8 \%)$ & $1.16(0.10-13.46)$ & 1.000 \\
\hline With fluoroquinolones & $1(4.3 \%)$ & $3(5.6 \%)$ & $0.77(0.08-7.84)$ & 1.000 \\
\hline Delayed tigecycline treatment & $6(26.1 \%)$ & $15(27.8 \%)$ & $0.92(0.30-2.77)$ & 0.879 \\
\hline
\end{tabular}

Abbreviations: $O R$ odd ratio, $C l$ confidence interval, MDRAB multi-drug-resistant Acinetobacter baumannii

${ }^{a}$ Categorical data are $\mathrm{n}(\%)$ of subject, continuous data are expressed as mean (standard deviation)

Table 6 Multivariate analysis of the predictors for the 30-day mortality

\begin{tabular}{lll}
\hline Variables & Adjusted OR (95\% Cl) & $p$ \\
\hline CURB65 score & $3.18(1.44-6.99)$ & 0.004 \\
Mechanical ventilation & $6.38(1.16-35.23)$ & 0.034 \\
Bacteremia & $21.00(0.99-443.40)$ & 0.050 \\
Duration of Tigecycline treatment & $0.97(0.87-1.08)$ & 0.567 \\
Tigecycline resistance & $0.20(0.05-0.78)$ & 0.021 \\
\hline
\end{tabular}

Abbreviations: $O R$ odd ratio, $\mathrm{Cl}$ confidence interval, MDRAB multidrugresistant Acinetobacter baumannii and $81.8 \%(27 / 33)$ were intermediate. High rate of resistant to tigecycline have also been reported previously in Asia. Although the mechanism of tigecycline resistance has not been identified, Park [25] showed that previously receiving carbapenems is an independent risk factor associated with the development of MDRAB resistant to tigecycline. Similarly, most patients in the study $(70.1 \%$, $54 / 77$ ) had previously received carbapenems. Surprisingly, only 8 patients had tigecycline-resistant MDRAB after tigecycline use, and it is consistent with other studies. This study is consistent with previous studies which suggest that previous exposure to a carbapenem increases the risk of Acinetobacter spp. resistant to tigecycline [26-28]. There are several limitations in this study. First, it was a single-center study. Although only 
monomicrobial MDRAB pneumonia was included in the study, the results may not be generalized in other infections and to all settings. Second, this is a retrospective study. The data collection from patient medical records may not be complete.

\section{Conclusion}

Tigecycline is a potential option for the treatment of nosocomial pneumonia infection caused by MDRAB, and to use it appropriately, factors such as the susceptibility of tigecycline to the isolate MDRAB should be considered, as the effect of tigecycline may not be effective when the $\mathrm{MIC}>2 \mathrm{mg} / \mathrm{L}$. In addition, the predictors for 30-day mortality are high CURB65 scores, higher resistance rate to tigecycline in the hospital, VAP and bacteremia caused by MDRAB, and shorter duration of tigecycline treatment.

\section{Abbreviations \\ Cl: Confidence interval; CLSI: Clinical and Laboratory Standards Institute; COPD: Chronic obstructive pulmonary disease; CRP: C-reactive protein; HAP: Hospital-acquired pneumonia; MDRAB: multidrug resistent Acinetobacter baumannii; Ors: Odd ratios; PCT: Procalcitonin; VAP: Ventilator associated pneumonia \\ Acknowledgements \\ We thank Dr. Jianquan Luo and Dr. Jian Qu for the statistical technical assistance. \\ Funding \\ This work was financially supported by Hunan Provincial Natural Science Foundation of China (2018JJ3771) and the foundation for excellence in youth scholars of the department of pharmacy, and these funding bodies didn't involve in the design of the study and collection, analysis, and interpretation of data and in writing the manuscript.}

\section{Availability of data and materials}

The data sets used and/or analyzed during the current study are available from the corresponding author on reasonable request.

\section{Access to the medical data}

Permission was granted by the Second Xiang Hospital, Central South University to have the access to the medical data for this research project.

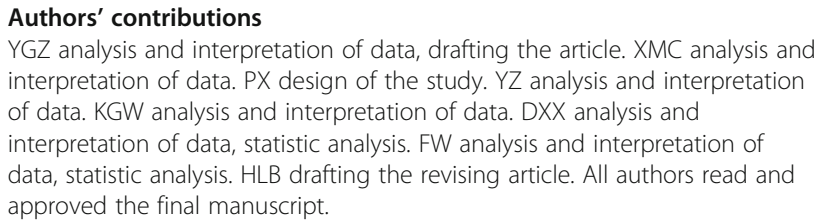

YGZ analysis and interpretation of data, drafting the article. XMC analysis and interpretation of data. PX design of the study. $Y Z$ analysis and interpretation of data. KGW analysis and interpretation of data. DXX analysis and interpretation of data, statistic analysis. FW analysis and interpretation of data, statistic analysis. HLB drafting the revising article. All authors read and approved the final manuscript.

\section{Ethics approval and consent to participate}

The study obtained consent from all patients and Ethics approval from the Second Xiangya Hospital of Central South University (Changsha, China).

\section{Consent for publication}

Not applicable.

\section{Competing interests}

The authors declare that they have no competing interests.

\section{Publisher's Note}

Springer Nature remains neutral with regard to jurisdictional claims in published maps and institutional affiliations.

\section{Author details}

'Department of Pharmacy, the Second Xiangya Hospital, Central South University, No.139 Middle Renmin Road, Changsha, Hunan 410011, People's Republic of China. ${ }^{2}$ Institute of Clinical Pharmacy, Central South University, Changsha, People's Republic of China. ${ }^{3}$ The Traditional Chinese Medicine hospital of Longhui, Changsha, Hunan, People's Republic of China.

${ }^{4}$ Department of Family Medicine, University of Alberta, Edmonton, Canada.

Received: 17 October 2018 Accepted: 10 April 2019

Published online: 25 April 2019

\section{References}

1. Tsioutis C, Kritsotakis El, Karageorgos SA, et al. Clinical epidemiology, treatment and prognostic factors of extensively drug-resistant Acinetobacter baumannii ventilator-associated pneumonia in critically ill patients. Int J Antimicrob Agents. 2016;48(5):492-7.

2. Kengkla K, Kongpakwattana K, Saokaew S, et al. Comparative efficacy and safety of treatment options for MDR and XDR Acinetobacter baumannii infections: a systematic review and network meta-analysis. J Antimicrob Chemother. 2018;73(1):22-32.

3. MacGowan AP. Tigecycline pharmacokinetic/pharmacodynamic update. J Antimicrob Chemother. 2008;62(Suppl1):111-6.

4. Tasina $E$, Haidich AB, Kokkali S, et al. Efficacy and safety of tigecycline for the treatment of infectious diseases: a meta-analysis. Lancet Infect Dis. 2011;11(11):834-44.

5. Ni W, Han Y, Zhao J, et al. Tigecycline treatment experience against multidrug-resistant Acinetobacter baumannii infections: a systematic review and meta-analysis. Int J Antimicrob Agents. 2016;47(2):107-16.

6. Chuang YC, Cheng CY, Sheng WH, et al. Effectiveness of tigecycline-based versus colistin-based therapy for treatment of pneumonia caused by multidrug-resistant Acinetobacter baumannii in a critical setting: a matched cohort analysis. BMC Infect Dis. 2014;14:102.

7. Cheng A, Chuang YC, Sun HY, et al. Excess mortality associated with ColistinTigecycline compared with Colistin-Carbapenem combination therapy for extensively drug-resistant Acinetobacter baumannii bacteremia: a multicenter prospective observational study. Crit Care Med. 2015;43(6):1194-204.

8. Jean SS, Hsieh TC, Hsu CW, et al. Comparison of the clinical efficacy between tigecycline plus extended-infusion imipenem and sulbactam plus imipenem against ventilator-associated pneumonia with pneumonic extensively drug-resistant Acinetobacter baumanniibacteremia, and correlation of clinical efficacy with in vitro synergy tests. J Microbiol Immunol Infect. 2016;49(6):924-33.

9. $\mathrm{Xu} \mathrm{L}$, Wang $Y \mathrm{~L}$, Du S, et al. Efficacy and safety of Tigecycline for patients with hospital-acquired pneumonia. Chemotherapy. 2015:16(61):323-30.

10. American Thoracic Society. Infectious diseases society ofAmerica. Guidelines for the management of adults with hospital-acquired, ventilator-associated, and health care associated pneumonia. Am J Respir Crit Care Med. 2005;171:388-416.

11. Rhee C. Using Procalcitonin to guide antibiotic therapy. Open Forum Infect Dis. 2017:4(1):1-10.

12. Ye JJ, Lin HS, Kuo AJ, et al. The clinical implication and prognostic predictors of tigecycline treatment for pneumonia involving multidrugresistant Acinetobacter baumannii. J Infect. 2011;63:351-61.

13. Clinical and Laboratory Standards Institute: Performance standards for antimicrobial susceptibility testing: 26th informational supplement, M100S17. Wayne, PA: CLSI; 2016

14. Mylotte JM, Naughton B, Saludades C, et al. Validation and application of the pneumonia prognosis index to nursing home residents with pneumonia. J Am Geriatr Soc. 1998;46:1538-44.

15. Tasbakan MS, Pullukcu H, Sipahi OR, et al. Is tigecyclin a good choice in the treatment of multidrug-resistant Acinetobacter baumannii pneumonia? J Chemother. 2011;23(6):345-9.

16. Lee YT, Tsao SM, Hsueh PR. Clinical outcomes of tigecycline alone or in combination with other antimicrobial agents for the treatment of patients with healthcare-associated multidrug-resistant Acinetobacter baumannii infections. Eur J Clin Microbiol Infect Dis. 2013;32(9):1211-20.

17. Schafer JJ, Goff DA, Stevenson KB, et al. Early experience with tigecycline for ventilator-associated pneumonia and bacteremia caused by multidrugresistant Acinetobacter baumannii. Pharmacotherapy. 2007:27(7):980-7.

18. Curcio D, Fernández F, Vergara J, et al. Late onset ventilator-associated pneumonia due to multidrug-resistant Acinetobacter spp.: experience with tigecycline. J Chemother. 2009;21(1):58-62. 
19. Weiss E, Essaied W, Adrie C, et al. Treatment of severe hospital-acquired and ventilator-associated pneumonia: a systematic review of inclusion and judgment criteria used in randomized controlled trials. Crit Care. 2017;21:162.

20. Wu X, Zhu Y, Chen Q, et al. Tigecycline therapy for nosocomial pneumonia due to Carbapenem-resistant gram-negative Bacteria in critically ill patients who received inappropriate initial antibiotic treatment: a retrospective case study. Biomed Res Int. 2016;2016:1-7.

21. Kim WY, Moon JY, Huh JW, et al. Comparable efficacy of Tigecycline versus Colistin therapy for multidrug-resistant and extensively drug-resistant Acinetobacter baumannii pneumonia in critically ill patients. PLoS One. 2016;11(3):e0150642.

22. Ulldemolins M, Roberts JA, Rello J, et al. The effects of Hypoalbuminaemia on optimizing antibacterial dosing in critically ill patients. Clin Pharmacokinet. 2011;50(2):99-110.

23. Wong G, Briscoe S, Adnan $\mathrm{S}$, et al. Protein binding of $\beta$-lactam antibiotics in critically ill patients: can we successfully predict unbound concentrations? Antimicrob Agents Chemother. 2013;57(12):6165-70.

24. Bhavnani SM, Rubino CM, Hammel JP, Cooper CA, et al. Pharmacological and patient-specific response determinants in patients with hospitalacquired pneumonia treated with Tigecycline. Antimicrob Agents Chemother. 2012;56(2):1065-72.

25. Park GE, Kang $\mathrm{Cl}$, Cha MK, et al. Bloodstream infections caused by Acinetobacter species with reduced susceptibility to tigecycline: clinical features and risk factors. Int J Infect Dis. 2017:62:26-31.

26. Lee $\mathrm{JY}$, Kang $\mathrm{Cl}$, Ko JH, et al. Clinical features and risk factors for development of breakthrough gram-negative bacteremia during Carbapenem therapy. Antimicrob Agents Chemother. 2016;60(11):6673-8.

27. Wang L, Wang YJ, Liu YY, et al. In vitro potential of Lycosin-I as an alternative antimicrobial drug for treatment of multidrug-resistant Acinetobacter baumanniiinfections. Antimicrob Agents Chemother. 2014; 58(11):6999-7002.

28. Zhang $T$, Wang $M$, Xie $Y$, et al. Active efflux pump adeB is involved in multidrug resistance of Acinetobacter baumannii induced by antibacterial agents. Exp Ther Med. 2017;13(4):1538-46.

Ready to submit your research? Choose BMC and benefit from:

- fast, convenient online submission

- thorough peer review by experienced researchers in your field

- rapid publication on acceptance

- support for research data, including large and complex data types

- gold Open Access which fosters wider collaboration and increased citations

- maximum visibility for your research: over $100 \mathrm{M}$ website views per year

At $\mathrm{BMC}$, research is always in progress.

Learn more biomedcentral.com/submissions 